\title{
Optimization of the TRAP assay to evaluate specificity of telomerase inhibitors
}

\author{
Kamilla Piotrowska ${ }^{1, *}$, Elke Kleideiter ${ }^{1, *}$, Thomas E Mürdter ${ }^{1}$, Sebastian Taetz ${ }^{2}$, \\ Christiane Baldes ${ }^{2}$, Ulrich Schaefer ${ }^{2}$, Claus-Michael Lehr ${ }^{2}$ and Ulrich Klotz ${ }^{1}$ \\ ${ }^{1}$ Dr Margarete Fischer-Bosch Institute of Clinical Pharmacology, Stuttgart, Germany and ${ }^{2}$ Department \\ of Biopharmaceutics and Pharmaceutical Technology, Saarland University, Saarbrücken, Germany
}

\begin{abstract}
Telomerase inhibition represents a promising approach to anticancer treatment. In order to clarify the therapeutic potential of telomerase inhibitors we examined different substances (small molecule compounds BIBR1532 and BRAC019, as well as hTR antisense oligonucleotides 2'-O-methyl RNA and PNA) in A-549, MCF-7, and Calu-3 cell lines in a cell-free TRAP assay. We demonstrated that each of the tested agents inhibited telomerase in all used cell lines and that the antisense oligonucleotides represent the most potent inhibitors. Interestingly, upon evaluating the specificity of telomerase inhibitors we found out that not all agents acted specifically against telomerase. We observed that BRACO19 and PNA had an inhibitory effect also on PCR amplification of the TSR8 oligonucleotide which is provided in the TRAP ${ }_{E Z E}^{R}$ kit as a PCR control. By modifying the experimental protocol and using a different reverse primer we were able to enhance PNA selectivity, although the PCR inhibition of the TSR8 control template by BRACO19 could not be prevented. We propose an explanation for the lack of target specificity and suggest caution when testing putative telomerase inhibitors, as it appears that some of those substances may not affect specifically telomerase or telomeric G-rich sequences and thus can lead to the misinterpretation of experimental results.
\end{abstract}

Laboratory Investigation (2005) 85, 1565-1569. doi:10.1038/labinvest.3700352; published online 19 September 2005

Keywords: telomerase; telomerase inhibitors; TRAP assay

Telomerase is an RNA-dependent DNA polymerase that synthesizes chromosomal DNA ends called telomeres consisting in man of the repetitive, sixbase motif (TTAGGG) ${ }_{n}$ and comprising 10-15 kb. The holoenzyme contains the catalytic subunit human telomerase reverse transcriptase (hTERT), the RNA template subunit, hTR, and several other accessory proteins. ${ }^{1}$ Progressive telomere shortening between 50 and $200 \mathrm{bp}$ occurs in the absence of telomerase in somatic cells at each cell division until a critical telomeric length is reached. ${ }^{2}$ Telomerase expression is switched off after embryonic differentiation in most normal cells (exceptions are germ cells, lymphocytes and stem cells), however, it is expressed in over $85 \%$ of tumors. ${ }^{2-5}$ Therefore, telomerase provides a promising novel molecular target for cancer treatment. ${ }^{1,6}$ There are numerous approaches towards the inhibition of telomerase, such as direct binding of non-nucleosidic com-

Correspondence: K Piotrowska, Dr Margarete Fischer-Bosch Institute of Clinical Pharmacology, Auerbachstraße 112, D-70376 Stuttgart, Germany.

E-mail: kamilla.piotrowska@ikp-stuttgart.de

* These authors contributed equally to this work.

Received 18 July 2005; revised 4 August 2005; accepted 8 August 2005; published online 19 September 2005 pounds like BIBR1532 to the catalytic hTERT component. ${ }^{7}$ Preventing the enzyme from interacting with telomeres is another strategy which can be accomplished by G-quadruplex-interactive compounds like BRACO19. ${ }^{8}$ In addition, the antisense approach using modified short RNA and DNA molecules such as RNA oligomers with methylsubstituted ribose sugar rings (2'-O-methyl RNA) and peptide nucleic acids (PNAs) directed against hTR can be applied to inhibit telomerase. ${ }^{9}$

We used the modified TRAP (telomerase repeat amplification protocol) assay ${ }^{10}$ to determine the influence of these substances on telomerase activity in vitro. We have tested telomerase inhibitors in cell lysates of A-549 (human non-small-cell lung carcinoma) cells to determine the minimal concentrations needed for inhibition as our research project focuses primarily on lung cancer. This malignancy causes most of the cancer fatalities and represents still a therapeutic challenge. In trying to generalize our findings we also confirmed that these concentrations effectively inhibited telomerase in two other widely used cell lines: Calu-3 (human lung adenocarcinoma), and MCF-7 (human breast adenocarcinoma). Moreover, we demonstrate that telomerase inhibitors may nonspecifically target telomerase or telomeric G-rich sequences and this effect is not 
restricted to one group of substances. The experimental results presented in this study may help to design more effective screening programs for potential cancer therapeutics based on the inhibition of telomerase.

\section{Materials and methods}

\section{Cell Lines}

A-549 cells (DSMZ, Braunschweig, Germany) were cultivated in Roswell Park Memorial Institute (RPMI) 1640 medium (Biochrom, Berlin, Germany) supplemented with 10\% FCS (Biochrom), $100 \mathrm{U} / \mathrm{ml}$ penicillin, $100 \mu \mathrm{g} / \mathrm{ml}$ streptomycin (Gibco BRL, Karlsruhe, Germany), and glutamine ( $2 \mathrm{mM}$, Biochrom) in $5 \% \mathrm{CO}_{2}$ at $37^{\circ} \mathrm{C}$. Calu-3 cells (HTB-55, ATCC, Manassas, VA, USA) were cultivated in Minimum Essential Medium (MEM) with Earl's Salts and L-glutamine (Gibco, Paisley, Scotland, UK) supplemented with 10\% FCS (Gibco), 1\% MEM nonessential amino acid solution (SigmaAldrich Chemie GmbH, Steinheim, Germany) and $55 \mathrm{mg} / 500 \mathrm{ml}$ sodium pyruvate (Sigma-Aldrich, cell culture tested) in $5 \% \mathrm{CO}_{2}$ at $37^{\circ} \mathrm{C}$.

\section{Telomerase Inhibitors}

Stock solutions of BIBR1532 (in DMSO) and BRACO19 (in sterile water) were aliquoted and stored at $-20^{\circ} \mathrm{C}$. $2^{\prime}$-O-methyl RNA inhibitor with a phosphorothioate (ps) backbone (5'-2'-O-methyl [C(ps)A(ps) GUUAGGGUU(ps)A(ps)G]-3', MWG-Biotech AG, Ebersberg, Germany) and 2'-O-methyl RNA mismatch $\quad\left(5^{\prime}-2^{\prime}-O\right.$-methyl[C(ps)A(ps)GUUAGAAUU (ps)A(ps)G]-3', MWG-Biotech AG) were dissolved in sterile water and stored at $-20^{\circ} \mathrm{C}$. PNA inhibitor (CAGTTAGGGTTAG, Applied Biosystems, Weiterstadt, Germany) and PNA mismatch (AAGTTAGA CAA, Applied Biosystems) were dissolved in sterile water and stored at $4^{\circ} \mathrm{C}$. PNAs are listed amino to carboxyl termini and contain carboxy-terminal lysine residues.

\section{Sample Preparation}

In all, $10^{6}$ cells of each sample were resuspended in $200 \mu$ l of 3-[(3-cholamidopropyl)dimethyl-ammonio]1-propanesulfonate (CHAPS) lysis buffer and incubated for $30 \mathrm{~min}$ on ice. After the incubation, lysates were centrifuged at $12000 \mathrm{rpm}$ for $20 \mathrm{~min}$ at $4^{\circ} \mathrm{C}$. The supernatant was recovered and protein concentrations were measured using the Biciuchoriuic acid (BCA) test (Sigma Aldrich Company, Deisenhofen, Germany).

\section{TRAP Assay}

Telomerase activity in cell lysates was measured as previously described ${ }^{11}$ by TRAP assay using the
TRAP ${ }_{\text {EZE }}^{\circledR}$ Telomerase Detection Kit (Chemicon International, Hampshire, UK) according to the manufacturer's protocol with the following modifications: oligonucleotide substrate (5'-AATCCGTCGAGCA GAGTT-3') (TS Primer, MWG-Biotech AG) labeled with an infrared dye (Carl Roth $\mathrm{GmbH}$, Karlsruhe, Germany) was used instead of the ready-to-use TS primer provided in the TRAPEZE kit in order to detect the PCR products using a DNA sequencer (Model 4000, LI-COR Biosciences GmbH, Bad Homburg, Germany). CX primer 5'-CCCTTACCCT TACCCTTACCCTAA-3 ${ }^{\prime 9}$ (Carl Roth GmbH, Karlsruhe, Germany) was used in the concentration of $30 \mu \mathrm{M}$ instead of RP primer (provided in the TRAP ${ }_{\text {EZE }}^{\mathbb{R}}$ kit as component of the primer mix) to increase the inhibitor target specificity. Also, $10 \mu \mathrm{l}$ of solution Q (Qiagen, Hilden, Germany) for better amplification was added to each reaction. Telomerase inhibitors were directly added to the master mix. The optimal results for all tested cell lines were achieved using $0.05 \mu \mathrm{g}$ protein per reaction.

After a 30 -min incubation at $30^{\circ} \mathrm{C}$, the samples were placed in PTB Thermal Cycler (Lab-Line, CLF, Emersacker, Germany) for 2 min at $94^{\circ} \mathrm{C}$, followed by 35 PCR cycles of denaturation at $94^{\circ} \mathrm{C}$ for $30 \mathrm{~s}$ and synthesis at $57^{\circ} \mathrm{C}$ for $30 \mathrm{~s}$ as well as a single postsynthesis step at $57^{\circ} \mathrm{C}$ for $30 \mathrm{~s}$. The PCR products were separated by electrophoresis on $6 \%$ nondenaturing polyacrylamide gels and detected using DNA sequencer. TSR8 and CHAPS lysis buffer (provided in the TRAP ${ }_{\text {EZE }}^{\mathbb{R}}$ kit) were used as positive and negative controls, respectively.

\section{Results}

We tested different concentrations of BIBR1532, BRACO19, 2'-O-methyl RNA, and PNA on A-549 cell lysates to determine the concentrations needed to significantly inhibit telomerase. As an example data are shown for BRACO19 in Figure 1. Effective inhibitory concentrations were as follows: BIBR1532: 5-20 $\mu \mathrm{M}$, BRACO19: 0.5-1 $\mu \mathrm{M}, \quad 2^{\prime}-O-$ methyl RNA: $0.1-1 \mu \mathrm{M}$, and PNA: $0.01-0.1 \mu \mathrm{M}$ (Figure 2). Application of mismatch variants of 2'-O-methyl RNA and PNA to the cell lysates did not result in telomerase inhibition (Figure 2). It should be noted that the $36 \mathrm{bp}$ internal control band was present in all samples indicating that the PCR was carried out properly. The internal control band is generated by primer $\mathrm{K} 1$ and a template TSK1 without the contribution of the reverse primer (RP, provided in the TRAP ${ }_{\mathrm{EZE}}^{\mathrm{R}}$ kit). In order to determine whether the inhibitory concentrations tested in A-549 cells are sufficient to effectively inhibit telomerase in other cell lines we applied the compounds to the cell lysates of the commonly used Calu-3 and MCF-7 cells. All tested substances inhibited telomerase in Calu-3 and MCF-7 cell lysates at the same concentrations as in A-549 cells (data not shown). Interestingly, when adding PNA 


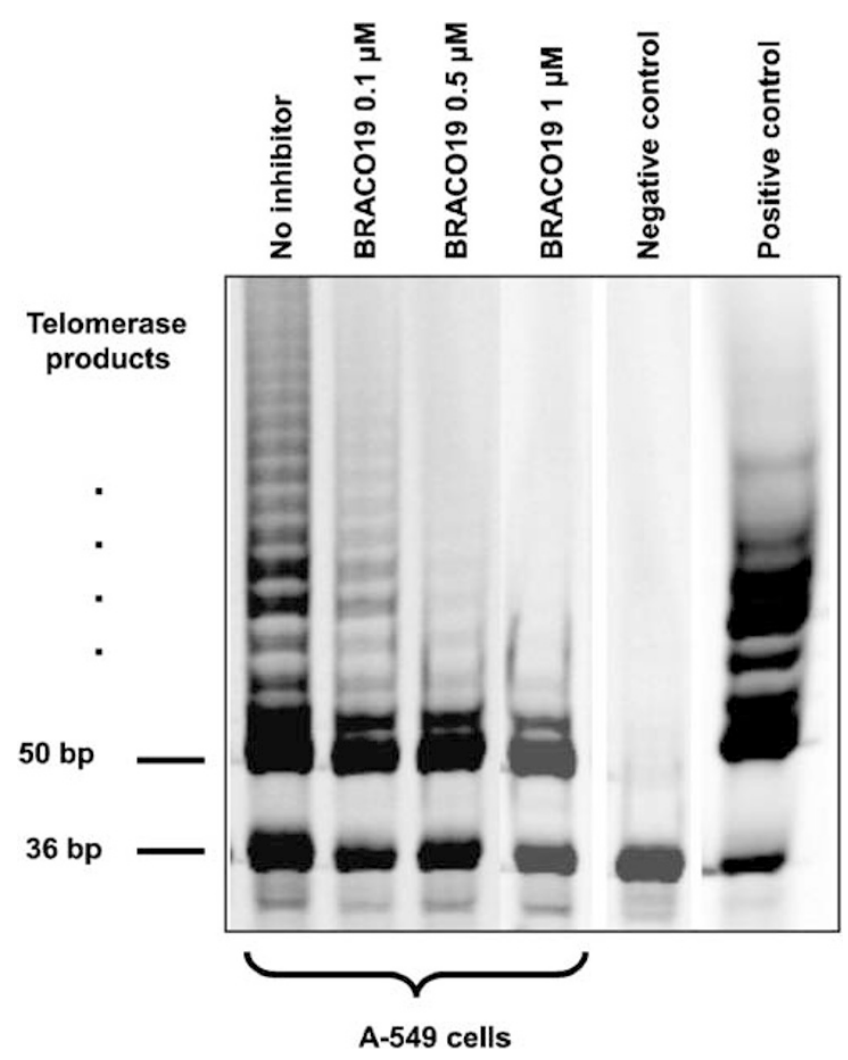

Figure 1 Concentration-dependent inhibition of telomerase by BRACO19 in A-549 cells. The lane denoted No inhibitor represents control A-549 cells to which no inhibitor was added. Negative control: CHAPS lysis buffer. Positive control: TSR8. $36 \mathrm{bp}$ band represents the internal standard.

and BRACO19 to the TSR8 internal control an inhibition of the PCR (the second step of the TRAP assay) was also observed (Figure 3). This methodological problem of specificity was addressed by modifying the analytical conditions in such a way that the primer mix provided in the TRAP ${ }_{\mathrm{EZE}}^{\mathbb{R}}$ kit was replaced by a different reverse primer (CX). In contrast to the unknown reverse primer RP from the TRAP ${ }_{\mathrm{EZE}}^{\mathbb{R}}$ kit sequence and concentration of the CX primer are known and the optimal concentration of the CX primer was determined $(30 \mu \mathrm{M})$. The efficiency of the detection of telomerase activity in A-549, MCF-7 or Calu-3 cells using CX primer was the same as in the experiments with the use of RP primer (Figure 4). As expected, the modified experimental conditions prevented the generation of the $36 \mathrm{bp}$ band. However, we observed no inhibition of TSR8 amplification with $0.05 \mu \mathrm{M}$ PNA as opposed to the previous experiments with the use of the RP primer, although $1 \mu \mathrm{M}$ BRACO19 was still able to induce strong PCR inhibition of TSR8 (Figure 5).

\section{Discussion}

As telomerase activity is mainly characterized by the TRAP assay we were interested in getting more information concerning the specificity of inhibition

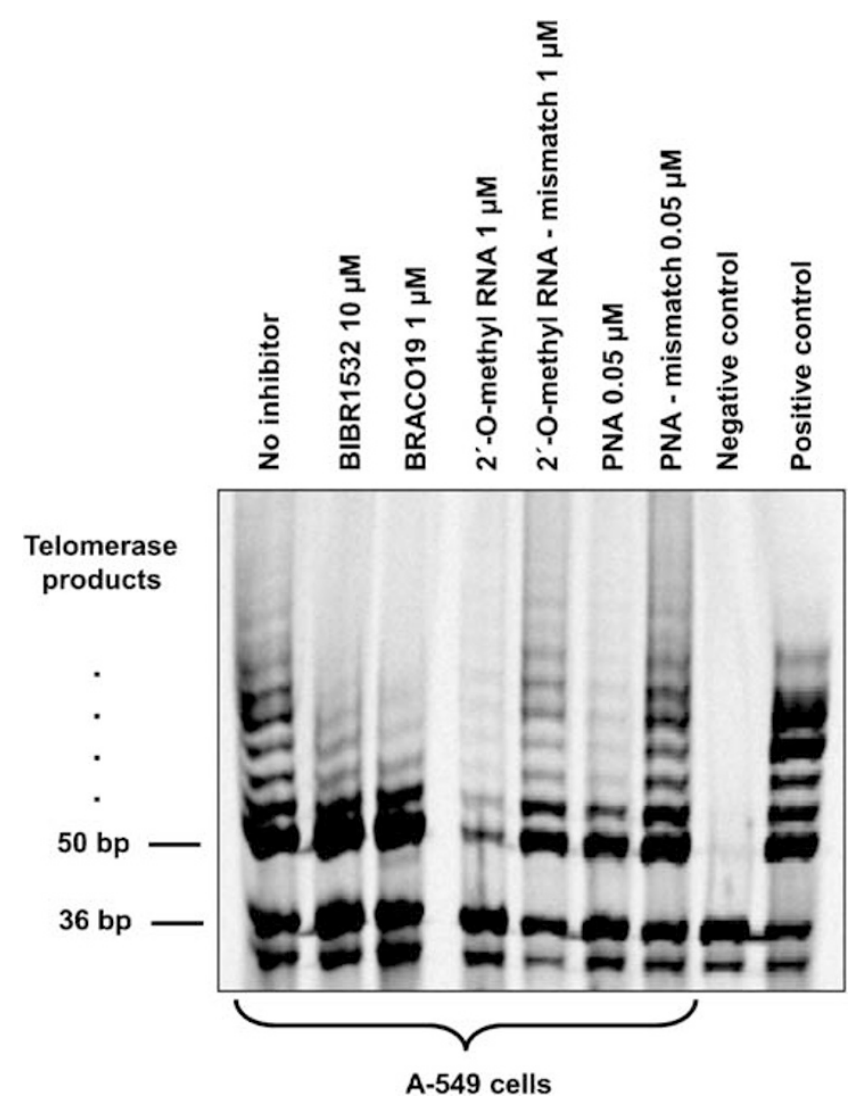

Figure 2 TRAP assay showing inhibition of telomerase activity by BIBR1532, BRACO19, 2'-O-methyl RNA, and PNA on telomerase in A-549 cells. $0.05 \mu \mathrm{g}$ protein per reaction was used. The lane denoted No inhibitor represents control A-549 cells to which no inhibitor was added. Negative control: CHAPS lysis buffer. Positive control: TSR8. $36 \mathrm{bp}$ band represents the internal standard.

in this widely applied method. Norton et $a l^{9}$ reported previously that PNA tested in the TRAP assay would hybridize to the reverse primer CX and thereby mimicing an inhibition of telomerase. However, the authors concluded that the hybridization could not account for the inhibition of telomerase as the reverse primer CX was in high excess relative to PNA. In contrast to Norton's group we have chosen telomerase-free reaction conditions to check the selectivity of the used compounds and applied each inhibitor to the TSR8 control template provided in the TRAP ${ }_{\mathrm{EZE}}^{\mathbb{R}}$ kit. TSR8 is an oligonucleotide identical to the TS primer extended with eight telomeric repeats AG(GGTTAG) 7 which can be used as a standard for quantitative analysis of TRAP products. Furthermore, detection of TSR8 indicates that the amplification step of the TRAP assay was performed effectively. The conditions for this experiment were the same as applied in testing telomerase inhibition in the selected cell lines. Interestingly, the results of this experiment revealed that the PCR inhibition of TSR8 manifested in a reduction of the number of bands on the gel was observed after applying $1 \mu \mathrm{M}$ BRACO19 and $0.05 \mu \mathrm{M}$ 


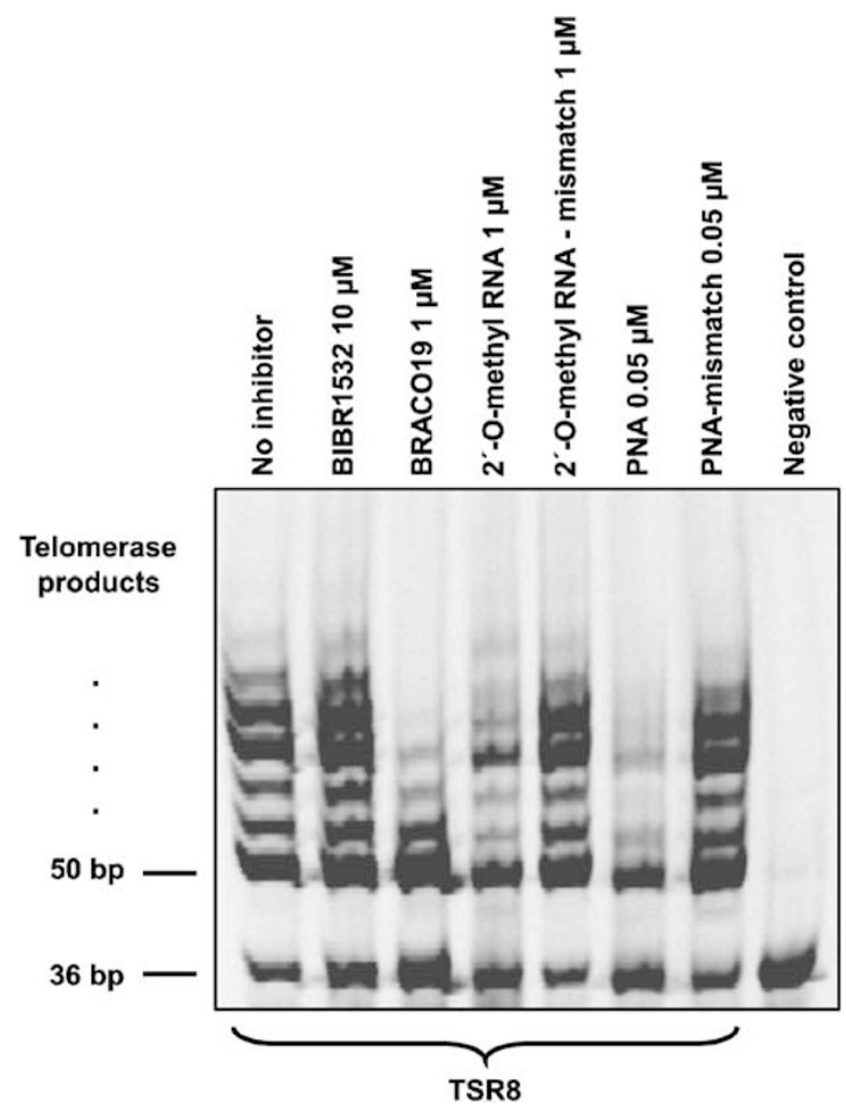

Figure 3 TRAP assay showing the inhibition of amplification of TSR8 positive control by BRACO19 and PNA. The lane denoted No inhibitor represents TSR8 to which no inhibitor was added. Negative control: CHAPS lysis buffer. $36 \mathrm{bp}$ band represents the internal standard.

PNA. Such inhibition of TSR8 amplification was not observed after applying $10 \mu \mathrm{M}$ BIBR1532, or $1 \mu \mathrm{M} 2^{\prime}$ $O$-methyl RNA (Figure 3). The presence of the $36 \mathrm{bp}$ band and simultaneous inhibition of TSR8 suggest that the inhibitors interact with the reverse primer RP which is needed for TSR8 amplification. Inhibition of TSR8 amplification by PNA in the TRAP assay using the standard primer mix provided in the TRAP ${ }_{\mathrm{EZE}}^{\mathbb{R}}$ kit (containing RP primer, K1 primer and TSK1 template) suggests PNA binds to the reverse primer RP. Such binding prevents successful amplification in the second step of the TRAP assay which is subsequently detected as reduced number of characteristic bands and is misleadingly interpreted as telomerase inhibition. We suggest that CX primer did not bind PNA in contrast to RP primer thus preventing from the inhibition of the second step (amplification) in the TRAP reaction. This enhances the PNA specificity and provides more accurate results.

As mentioned above $1 \mu \mathrm{M}$ BRACO19 was still able to inhibit the amplification of TSR8 which can be explained by its proposed mechanism of action: BRACO19 inhibits the access of telomerase by interacting with telomeric G-quadruplex DNA. ${ }^{8}$ It is very conceivable that BRACO19 recognizes TSR8

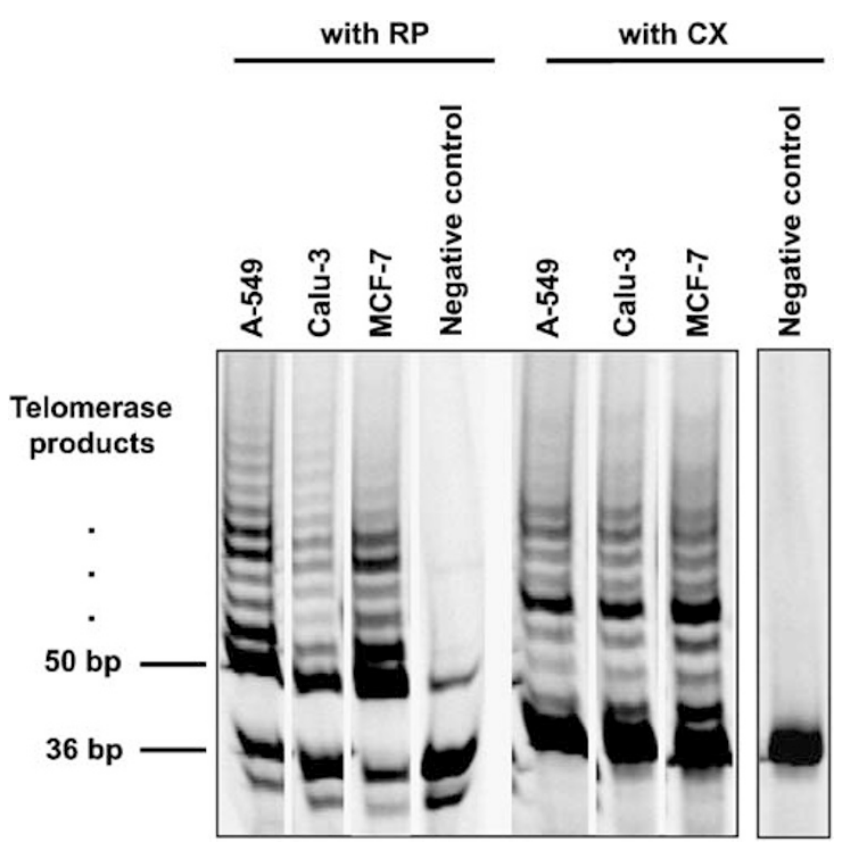

Figure 4 TRAP assay performed to compare two different experimental conditions: use of RP vs CX primer. The assay was performed for A-549, Calu-3, and MCF-7 cells. Upon amplification with CX primer no internal standard is generated. Negative control: CHAPS lysis buffer. $36 \mathrm{bp}$ band represents the internal standard.

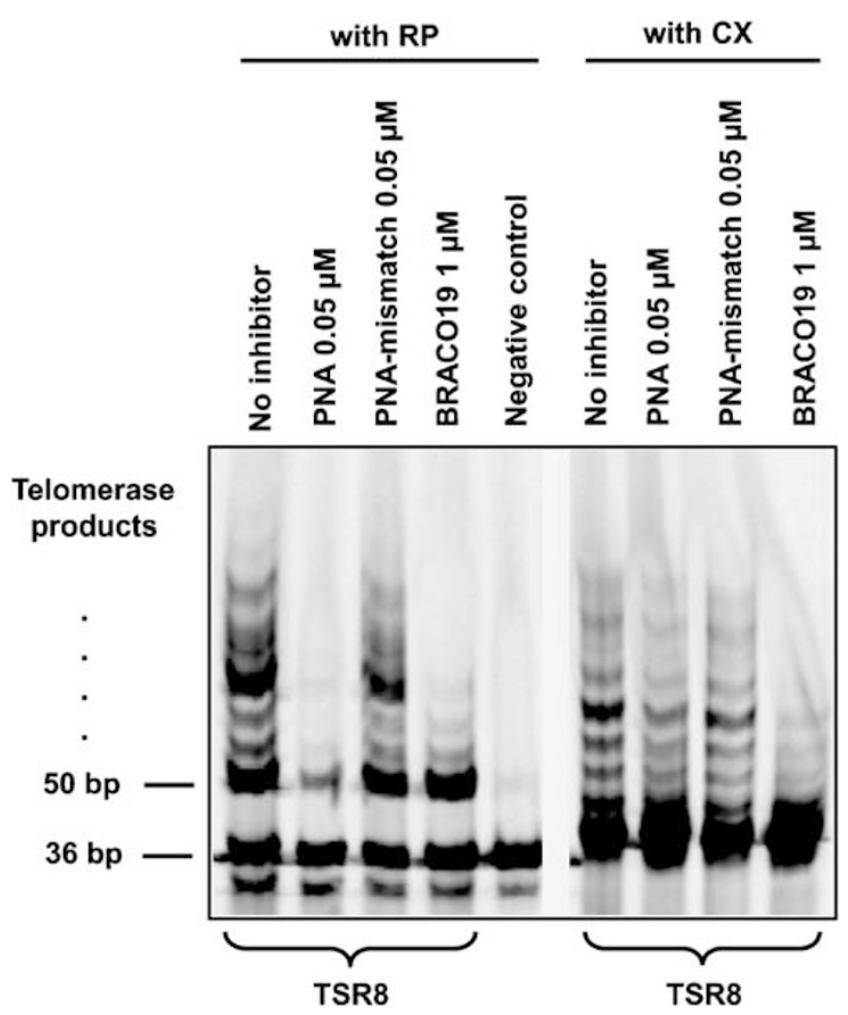

Figure 5 TRAP assay performed on TSR8 using either the standard primer RP or the alternative CX primer. The lane denoted No inhibitor represents TSR8 to which no inhibitor was added. Negative control: CHAPS lysis buffer. $36 \mathrm{bp}$ band represents the internal standard. 
G-rich sequence as a 'telomere-like' target and interacts with it which may disturb Taq polymerase action and/or prevent primer binding and causes PCR inhibition of TSR8. Therefore, introduction of the reverse primer CX does not have any effect on the inhibition induced by BRACO19, as it does not prevent BRACO19 from the interaction with TSR8. It cannot be excluded that BRACO19 may target nontelomeric G-rich sequences, and therefore its inhibitory effect on telomerase should be investigated carefully.

Our experiments with A-549 cells demonstrate that caution is recommended when testing putative telomerase inhibitors which subsequently are planned to be used in various cellular or in vivo models. We suggest that a test for PCR inhibition should be always performed by including TSR8 as a control when screening for putative telomerase inhibitors because otherwise experimental results can be misinterpreted due to the inhibition of the PCR reaction rather than the inhibition of telomerase. Since the TRAP assay is widely used and has been applied in studies with different tumor cells the outlined problem with its specific inhibition should be also considered when working with other cell types.

Further optimization of the TRAP assay is needed in order to exclude any potential unspecific interactions within this assay, so that the observed reduction of bands on the gel is based solely on selective telomerase inhibition.

\section{Acknowledgements}

We thank Jessica Lauser for her excellent technical assistance. BIBR1532 was synthesized by G Heinkele, Dr Margarete Fischer-Bosch Institute of Clinical Pharmacology, Stuttgart, Germany, and BRACO19 by Endotherm GmbH, Saarbrücken, Germany. MCF-7 cells were kindly provided by Dr M Buck, Dr Margarete Fischer-Bosch Institute of
Clinical Pharmacology, Stuttgart, Germany. The authors declare no duality of interest. The project is financially supported by German Cancer Aid (10-2035-Kl I), Bonn, Germany and Robert Bosch Foundation (I1-1/03), Stuttgart, Germany.

\section{References}

1 Helder MN, Wisman GB, van der Zee GJ. Telomerase and telomeres: from basic biology to cancer treatment. Cancer Invest 2002;20:82-101.

2 Janknecht R. On the road to immortality: hTERT upregulation in cancer cells. FEBS Lett 2004;564:9-13.

3 Harle-Bachor C, Boukamp P. Telomerase activity in the regenerative basal layer of the epidermis in human skin and in immortal and carcinoma-derived skin keratinocytes. Proc Natl Acad Sci USA 1996;93: 6476-6481.

4 Ramirez RD, Wright WE, Shay JW, et al. Telomerase activity concentrates in the mitotically active segments of human hair follicles. J Invest Dermatol 1997;108: 113-117.

5 Weng NP, Hathcock KS, Hodes RJ. Regulation of telomere length and telomerase in $\mathrm{T}$ and $\mathrm{B}$ cells: a mechanism for maintaining replicative potential. Immunity 1998;9:151-157.

6 Rezler EM, Bearss DJ, Hurley LH. Telomere inhibition and telomere disruption as processes for drug targeting. Annu Rev Pharmacol Toxicol 2003;43:359-379.

7 Damm K, Hemmann U, Garin-Chesa P, et al. A highly selective telomerase inhibitor limiting human cancer cell proliferation. EMBO J 2001;20:6958-6968.

8 Gowan SM, Harrison JR, Patterson L, et al. A G-quadruplex-interactive potent small-molecule inhibitor of telomerase exhibiting in vitro and in vivo antitumor activity. Mol Pharmacol 2002;61:1154-1162.

9 Norton JC, Piatyszek MA, Wright WE, et al. Inhibition of human telomerase activity by peptide nucleic acids. Nat Biotechnol 1996;14:615-619.

10 Kim NW, Piatyszek MA, Prowse KR, et al. Specific association of human telomerase activity with immortal cells and cancer. Science 1994;266:2011-2015.

11 Kleideiter E, Schwab M, Friedrich U, et al. Telomerase activity in cell lines of pediatric soft tissue sarcomas. Pediatr Res 2003;54:718-723. 